\title{
Efficacy and durability of multifactorial intervention on mortality and MACEs: a randomized clinical trial in type-2 diabetic kidney disease
}

Ferdinando Carlo Sasso ${ }^{1 *}$,, Pia Clara Pafundi ${ }^{1}$, Vittorio Simeon², Luca De Nicola ${ }^{1}$, Paolo Chiodini ${ }^{2}$, Raffaele Galiero', Luca Rinaldi ${ }^{1}$, Riccardo Nevola' ${ }^{1}$, Teresa Salvatore ${ }^{3}$, Celestino Sardu ${ }^{1}$, Raffaele Marfella', Luigi Elio Adinolfi' and Roberto Minutolo ${ }^{1}$ on behalf of NID-2 Study Group Investigators

\begin{abstract}
Background: Multiple modifiable risk factors for late complications in patients with diabetic kidney disease (DKD), including hyperglycemia, hypertension and dyslipidemia, increase the risk of a poor outcome. DKD is associated with a very high cardiovascular risk, which requires simultaneous treatment of these risk factors by implementing an intensified multifactorial treatment approach. However, the efficacy of a multifactorial intervention on major fatal/non-fatal cardiovascular events (MACEs) in DKD patients has been poorly investigated.

Methods: Nephropathy in Diabetes type 2 (NID-2) study is a multicentre, cluster-randomized, open-label clinical trial enrolling 395 DKD patients with albuminuria, diabetic retinopathy (DR) and negative history of CV events in 14 Italian diabetology clinics. Centres were randomly assigned to either Standard-of-Care (SoC) $(n=188)$ or multifactorial intensive therapy (MT, $n=207$ ) of main cardiovascular risk factors (blood pressure $<130 / 80 \mathrm{mmHg}$, glycated haemoglobin $<7 \%$, LDL, HDL and total cholesterol $<100 \mathrm{mg} / \mathrm{dL}$, $>40 / 50 \mathrm{mg} / \mathrm{dL}$ for men/women and $<175 \mathrm{mg} / \mathrm{dL}$, respectively). Primary endpoint was MACEs occurrence by end of follow-up phase. Secondary endpoints included single components of primary endpoint and all-cause death.

Results: At the end of intervention period (median 3.84 and 3.40 years in MT and SoC group, respectively), targets achievement was significantly higher in MT. During 13.0 years (IQR 12.4-13.3) of follow-up, 262 MACEs were recorded (116 in MT vs. 146 in SoC). The adjusted Cox shared-frailty model demonstrated 53\% lower risk of MACEs in MT arm (adjusted HR 0.47, 95\% Cl 0.30-0.74, $P=0.001$ ). Similarly, all-cause death risk was 47\% lower (adjusted HR 0.53, 95\%Cl $0.29-0.93, P=0.027)$.
\end{abstract}

Conclusion: MT induces a remarkable benefit on the risk of MACEs and mortality in high-risk DKD patients. Clinical Trial Registration ClinicalTrials.gov number, NCT00535925. https://clinicaltrials.gov/ct2/show/NCT00535925 Keywords: Diabetic nephropathy, Multifactorial intervention, Intensified treatment, CV risk factors, Very high CV risk, MACE

*Correspondence: ferdinandocarlo.sasso@unicampania.it

${ }^{1}$ Department of Advanced Medical and Surgical Sciences, University of Campania "Luigi Vanvitelli", Piazza Luigi Miraglia 2, 80138 Naples, Italy

Full list of author information is available at the end of the article

\section{Background}

Type 2 diabetes mellitus (T2DM) patients are at high risk of death, myocardial infarction (MI) and stroke compared to the general population [1]. More important, original author(s) and the source, provide a link to the Creative Commons licence, and indicate if changes were made. The images or other third party material in this article are included in the article's Creative Commons licence, unless indicated otherwise in a credit line to the material. If material is not included in the article's Creative Commons licence and your intended use is not permitted by statutory regulation or exceeds the permitted use, you will need to obtain permission directly from the copyright holder. To view a copy of this licence, visit http://creativecommons.org/licenses/by/4.0/. The Creative Commons Public Domain Dedication waiver (http://creativeco mmons.org/publicdomain/zero/1.0/) applies to the data made available in this article, unless otherwise stated in a credit line to the data. 
onset of diabetic kidney disease (DKD) remarkably worsens cardiovascular prognosis, as demonstrated by a very large meta-analysis showing that hazards for cardiovascular mortality at a given eGFR/albuminuria are higher among T2DM patients throughout the whole spectrum of disease [2]. The major role of DKD on cardiovascular outcome has also been supported by an observational study on 1.3 million of patients reporting a higher incidence rate of MI in DKD patients versus diabetes or chronic kidney disease alone [3]. Of note, this finding is enhanced by the coexistence of proteinuria; indeed, albuminuria and low eGFR per se accelerate atherosclerosis process. Such a marked cardiovascular risk significantly modifies the outcome of DKD patients who often do not survive long enough to reach the natural fate of end-stage kidney disease [3-6].

The importance of a multifactorial approach in T2DM has been emphasized by the analysis of Swedish National Diabetes Register comparing prognosis of $\sim 270,000$ T2DM patients versus $\sim 1,300,000$ age- and gendermatched controls [1]. In particular, T2DM patients with five risk-factors within target range showed either a small or any excess risk of death, MI or stroke, as compared with controls [1]. However, DKD was either mild or absent in the vast majority of patients (mean GFR $84 \mathrm{~mL} / \mathrm{min}$ and $4.9 \%$ with macroalbuminuria). Furthermore, achievement of targets for multiple risk factors was uncommon (5\%).

As for observational studies, also multi-target interventional trials in DKD are lacking. The Steno-2 is the only study evaluating the effects of multifactorial approach in 160 microalbuminuric patients. The trial disclosed a significant reduction of both mortality and cardiovascular risk after implementing an intensified approach to multiple targets $[7,8]$. However, Steno-2 was a singlecentre study, carried out in patients with preserved renal function (mean GFR $118 \mathrm{~mL} / \mathrm{min}$ ) and mostly without diabetic retinopathy (DR) (74\%), that is, a sign of microangiopathy strictly associated with cardiovascular risk besides being a recognized marker of DKD [9].

Aim of the present trial is to evaluate the efficacy of a multifactorial intensive therapy (MT) versus Standardof-Care (SoC) on major fatal and non-fatal cardiovascular events (MACEs) in a population with DKD patients with albuminuria and retinopathy. Durability of the effect of intensified treatment was tested by extending followup to several years after the end of intervention phase.

\section{Methods}

Trial design

The Nephropathy In Diabetes type 2 (NID-2) study is an open-label cluster randomized clinical trial in a population referred to 14 Italian diabetology clinics [10]. To maximize the contrast between the two approaches, we randomized clinics rather than patients. Indeed, in the latter modality of randomization similarities between the two interventions are expected to ensue over the long-term. Centres were randomly assigned to either MT therapy or SoC. A questionnaire ascertained that all participating physicians were well aware of the guidelines on T2DM management published at the time of the study [11-14].

All MACEs diagnoses were performed in each patient according to the diagnostic criteria defined by the international standards of care guidelines [15-17]. MACEs were evaluated by cardiologist blinded to the study arm (MT or SoC), either belonging to the same Centres or to hospitals where patients were referred for acute events.

\section{Participants and procedures}

We considered eligible T2DM patients with age $\geq 40$ years, persistent albuminuria $\geq 30 \mathrm{mg} / 24 \mathrm{~h}$ in at least two of three $24 \mathrm{~h}$-urine collections in the last 6 months), severe DR (according to the Wilkinson et al.) [18], diabetes onset at age $>30$ years, absence of neoplastic/psychiatric diseases and follow-up at the centre $\geq 12$ months. Exclusion criteria were previous MI or stroke, severe hepatic or cardiac failure.

Patients were enrolled between October 2005 and October 2008. The intervention phase was scheduled for a period of four years, and it was completed in December 2011. Then, patients were followed until May 2019 to achieve the number of events needed for the primary outcome.

The protocol was approved by the ethics committee of University of Campania "Luigi Vanvitelli" (clinicaltrials. gov: NCT00535925) and is in accordance with the 1976 Declaration of Helsinki and its later amendments. All participants signed their informed consent.

\section{Randomization}

All patients enrolled in each clinic were randomized, according to a cluster-randomization procedure, in two arms, MT and SoC. Randomization of centres was stratified based on their size, in order to reduce difference in the number of patients allocated to the two treatment arms.

The intensified therapy group was initiated to the therapeutic regimen summarized below and detailed and in Additional file 1: Appendix S1. Patients assigned to the conventional therapy group followed the therapy usually administered at their outpatient clinic; hence, they could receive any therapeutic change considered appropriate by their caregiver, under the respect of the good clinical practice rules. 


\section{Targets}

In either arm participating physicians were required to adhere to guideline-based clinical targets recommended at the time of study initiation: [11-14] (a) systolic blood pressure $(\mathrm{SBP})<130 \mathrm{mmHg}$, (b) diastolic blood pressure $(\mathrm{DBP})<80 \mathrm{mmHg}$, (c) glycated haemoglobin $(\mathrm{HbA} 1 \mathrm{c})<7 \%$, (d) fasting serum LDL cholesterol $<100 \mathrm{mg} / \mathrm{dL}$, (e) fasting serum HDL cholesterol $>40 / 50 \mathrm{mg} / \mathrm{dL}$ (for men/women, respectively), and (f) fasting total serum cholesterol $<175 \mathrm{mg} / \mathrm{dL}$.

\section{Study arms}

In SoC group, the subjects received the therapy usually administered at their diabetic outpatient for the management of blood pressure, glycaemic and lipid control, and antiplatelet treatment. During the study, these patients received all therapeutic modifications considered appropriate by their physician, in the respect of the good clinical practice.

In MT group, the patients were treated with prespecified algorithm for management of hypertension, glycol-metabolic control and dyslipidemia, including non-pharmacological and pharmacological treatment, as detailed in Additional file 1: Appendix 1. Briefly, specific recommendation for physical activity and low sodium diet were provided to patients in written form. In addition, renin-angiotensin system blockade was implemented by initial association of ACEi and ARBs with a strict monitoring of GFR and serum potassium, followed by stepwise addition of other anti-hypertensive drug classes. They received low-dose aspirin as primary prevention, unless contraindicated or not tolerated. Statin was added if non-pharmacological therapy was ineffective in reaching the target.

All patients, regardless of the study group, underwent control visits at their diabetes centre every six months to monitor laboratory and clinical parameters and compliance to therapies and lifestyle hints. During each visit, investigators carefully monitored the occurrence of adverse events. In MT group, additional visits could be planned if one or more risk factors resulted out of target. At each visit, adherence to pharmacological protocol as well as to lifestyle recommendations (see Additional file 1: Appendix S1) was strictly monitored and strengthened.

eGFR was estimated using the CKD-EPI equation and, since creatinine was not standardized, we reduced creatinine values by $5 \%$ [19].

\section{Outcomes}

Primary endpoint was a composite of fatal and non-fatal MACEs, including cardiovascular mortality, non-fatal MI (documented instrumentally and/or enzymatically), nonfatal stroke, coronary-artery by-pass, revascularization procedures (PTCA) and lower limbs major amputation, whichever occurred first. In both arms, all endpoints were captured and recorded by investigators in an electronic Case Report Form (CRF) at each visit.

Since the planned number of events was not reached during the initial 4-year time frame (interventional phase), incidence of the primary end point was assessed throughout the follow-up phase, that in the original study design was planned to assess the durability of effects of the intensified treatment.

During this extension phase, following the end of intervention, all patients enrolled in both arms were treated by their own physicians according to the good clinical practice.

As secondary endpoints, we considered each single component of primary endpoint, and all-cause death at the end of the follow-up phase, as well as MACEs and the achievement of BP, HbA1c and total, HDL and LDL cholesterol goals at the end of intervention phase.

\section{Sample size}

Study is powered to detect a Hazard Ratio (HR) of 0.67 in the comparison of the two groups, with an $80 \%$ power and a two-sided type I error of 5\%, assuming an intraclass correlation coefficient of 0.01 . For this purpose, with a sample size of about 420 patients, 14 overall clusters, an average of 30 subjects per cluster, and an expected surviving proportion of $30 \%$ at ten years in the SoC group, we determined a number of events needed of 258 .

\section{Statistical analysis}

All statistical analyses were performed after the end of the follow-up and the achievement of number of events needed for the analysis of the primary outcome. A statistical analysis plan was prepared before the central database was locked for final data extraction and analysis. Categorical data were expressed as number and percentage, while continuous variables as either median and interquartile range or mean and standard deviation, based on their distribution assessed by the Shapiro-Wilk test. In order to check for imbalance in cluster randomization, we compared variables at baseline by using the method proposed by Leyrat et al. [20] Standardized differences (SDiff) were calculated for continuous and dichotomous variable. P-values to take into account clustering were computed by generalized estimating equations (GEE) model with cluster as group variable [21]. Distribution of dependent variable and link function was used as appropriate (gaussian and identity for continuous variable, binomial and logit for dichotomous variable). Comparison of groups at end of intervention 
was performed applying the same methodology, further adjusting for baseline values as covariate.

Criteria on SDiff cut-offs reported by Leyrat et al. [20] were used to establish covariates imbalanced at baseline. Moreover, to evaluate a global imbalance, $c$-statistic was calculated performing a logistic model with treatment arm as dependent variable and selected baseline variables as covariate.

Median follow-up time was calculated by the inverse Kaplan-Meier procedure. The primary endpoint was analysed according to the intention-to-treat principle, with event curves for the time-to-first event based on Kaplan-Meier analysis. Cox regression model was used to calculate HR and 95\% Confidence Interval (CI). Due to the cluster randomized study design, a Cox sharedfrailty model was fitted. Across centres, the frailties are assumed to be gamma-distributed latent random effects affecting the hazard multiplicatively. In the univariate analysis, only treatment group was included as covariate. In the multivariable analyses, depending on imbalance detection of each variable (Leyrat method), association with the outcome of interest and evidence from the literature, we adjusted the Cox regression models for age, sex, SBP, haemoglobin, eGFR, albuminuria, HbA1c, total cholesterol, triglycerides (log-scaled), statins and antiplatelets therapy at baseline to reduce risk of bias. Data were analysed using STATA 16.0 software (StataCorp. 2019. College Station, TX: StataCorp LLC).

\section{Results}

\section{Patients}

Out of the 850 eligible patients originally enrolled in the NID2 cross-sectional study [10, 22], we randomized 395 patients (207 to MT arm and 188 to SoC arm). Flow chart is reported in Fig. 1. Intervention and $\mathrm{SoC}$ groups were mostly similar for baseline characteristics (Table 1). However, patients in the group $\mathrm{SoC}$ were slightly older than those randomized to MT arm. HbA1c goal was more prevalent among controls. All variables, except for creatinine, showed a value of SDiff higher than $5 \%$. These values are reported in Additional file 2: Fig. S1 to provide information on the direction (treatment arm) of the imbalance. Overall, SoC arm showed a more favourable baseline clinical picture compared with the intervention

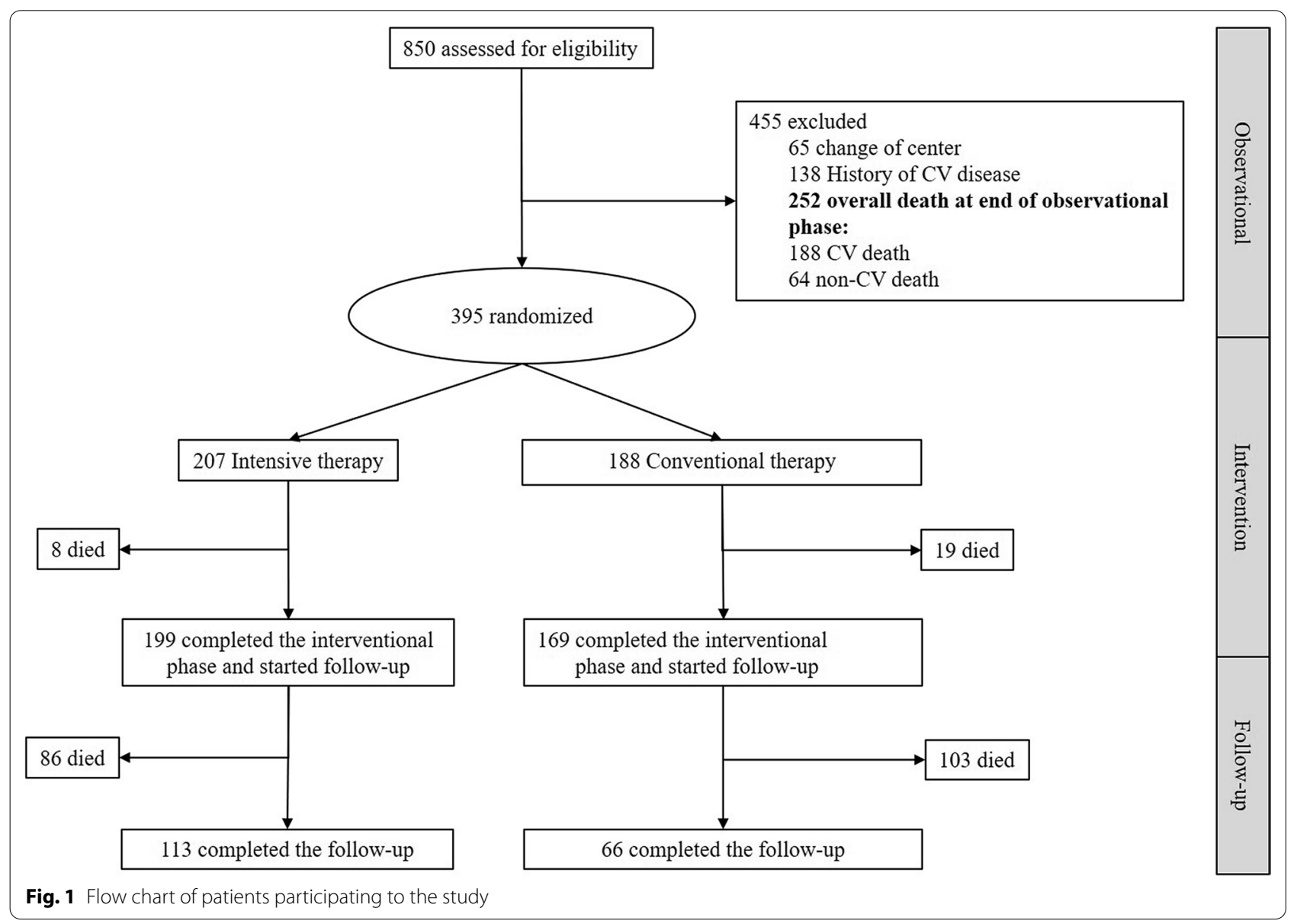


Table 1 Baseline characteristics of the study population $(n=395)$

\begin{tabular}{|c|c|c|c|c|}
\hline Parameter & SoC $(n=188)$ & Intervention $(n=207)$ & p & SDiff (\%) \\
\hline Male Sex, No. (\%) & $83(44.1)$ & $103(49.8)$ & 0.382 & 11.3 \\
\hline Age, mean (SD), y & $68.2(8.8)$ & $66.1(9)$ & 0.046 & 24.1 \\
\hline BMI $\left(\mathrm{kg} / \mathrm{m}^{2}\right)$, mean (SD) & $29.4(4.9)$ & $28.5(4.7)$ & 0.288 & 19.1 \\
\hline \multicolumn{5}{|l|}{ Blood pressure (mmHg), mean (SD) } \\
\hline Systolic & $134.7(12.6)$ & $133.8(14.3)$ & 0.791 & 6.1 \\
\hline Diastolic & $78.3(7.7)$ & $80.8(7.4)$ & 0.002 & -32.8 \\
\hline Systolic BP target, No. (\%) & $95(50.5)$ & $112(54.1)$ & 0.142 & -17.0 \\
\hline Diastolic BP target, No. (\%) & $150(79.8)$ & $136(65.7)$ & 0.274 & 19.2 \\
\hline Blood Pressure Target, No. (\%) & $90(47.9)$ & $105(55.3)$ & 0.193 & -14.8 \\
\hline Creatinine (mg/dL), mean (SD) & $1.16(0.5)$ & $1.17(0.5)$ & 0.564 & -1.4 \\
\hline eGFR EPI-CKD $\left(\mathrm{mL} / \mathrm{min} / \mathrm{m}^{2}\right)$, mean (SD) & $62.7(21.2)$ & $65.4(23)$ & 0.350 & -12.1 \\
\hline \multicolumn{5}{|l|}{ eGFR EPI-CKD stage, No. (\%) } \\
\hline 1 & $20(10.6)$ & $45(21.7)$ & 0.600 & -31.1 \\
\hline 2 & $80(42.6)$ & $77(37.2)$ & & \\
\hline 3 & $76(40.4)$ & $71(34.3)$ & & \\
\hline 4 & $10(5.3)$ & $11(5.3)$ & & \\
\hline 5 & $2(1.1)$ & $3(1.5)$ & & \\
\hline Albuminuria (mg/day), median [IQR] & $57.3[35-158.1]$ & $120.5[75.8-223.8]$ & 0.115 & -25.2 \\
\hline Haemoglobin (mg/dL), mean (SD) & $13.4(1.3)$ & $12.9(1.5)$ & 0.377 & 36.8 \\
\hline Glycemia (mg/dL), mean (SD) & $152.7(49)$ & $155.9(43.1)$ & 0.924 & -6.9 \\
\hline HbA1c (\%), mean (SD) & $7.3(1.1)$ & $7.5(1.1)$ & 0.345 & -18.2 \\
\hline HbA1c Target, No. (\%) & $100(53.2)$ & $66(31.9)$ & 0.001 & 43.1 \\
\hline Diabetes duration, median [IQR], y & $9(6-15)$ & $9(7-16)$ & 0.801 & -11.4 \\
\hline \multicolumn{5}{|l|}{ Cholesterol (mg/dL), mean (SD) } \\
\hline Total & $185.8(34.1)$ & $187.6(33.2)$ & 0.688 & -5.5 \\
\hline $\mathrm{LDL}$ & $111.5(30.8)$ & $107.5(27)$ & 0.703 & 13.7 \\
\hline Total Cholesterol Target, No. (\%) & $67(35.6)$ & $64(30.9)$ & 0.475 & 5.6 \\
\hline HDL Cholesterol Target, No. (\%) & $99(52.7)$ & $75(36.2)$ & 0.237 & 14.5 \\
\hline LDL Cholesterol Target, No. (\%) & $73(38.8)$ & $69(33.3)$ & 0.911 & 6.2 \\
\hline Triglycerides (mg/dL), median (IQR) & $111(88-153)$ & $148(115-190)$ & 0.063 & -42.7 \\
\hline \multicolumn{5}{|l|}{ Therapy } \\
\hline Anti-hypertensive Therapy, №. (\%) & & & 0.791 & -24.8 \\
\hline 1 & $105(55.9)$ & $94(45.4)$ & & \\
\hline 2 & $20(10.6)$ & $31(15)$ & & \\
\hline 3 & $32(17)$ & $44(21.3)$ & & \\
\hline 4 & $18(9.6)$ & $27(13)$ & & \\
\hline 5 & $13(6.9)$ & $11(5.3)$ & & \\
\hline \multicolumn{5}{|l|}{ ACEi/ARBs, No. (\%) } \\
\hline ACEi & $119(63.2)$ & $125(60.2)$ & 0.748 & -25.6 \\
\hline ARBs & $66(35.4)$ & $77(37.4)$ & & \\
\hline $\mathrm{ACE}+\mathrm{ARBS}$ & $3(1.4)$ & $5(2.4)$ & & \\
\hline Diuretics, No. (\%) & $83(44.1)$ & $113(54.6)$ & 0.840 & -21.0 \\
\hline Calcium Channel Blockers, No. (\%) & $63(33.5)$ & $82(39.6)$ & 0.792 & -12.7 \\
\hline Beta-blockers, No. (\%) & $31(16.5)$ & $38(18.4)$ & 0.655 & -4.9 \\
\hline Alpha-blockers, No. (\%) & $13(6.9)$ & $11(5.3)$ & 0.395 & 6.7 \\
\hline Diabetes Therapy, No. (\%) & & & 0.361 & -27.7 \\
\hline Diet & $16(8.5)$ & $5(2.4)$ & & \\
\hline Insulin & $50(26.6)$ & $60(29)$ & & \\
\hline Oral anti-hyperglycemics* & $97(51.6)$ & $108(52.2)$ & & \\
\hline
\end{tabular}


Table 1 (continued)

\begin{tabular}{llll}
\hline Parameter & SoC $(\mathbf{n}=\mathbf{1 8 8})$ & Intervention $(\mathbf{n = 2 0 7 )}$ & $\mathbf{p}$ \\
\hline Combined Therapy & $25(13.3)$ & $33(15.9)$ & SDiff (\%) \\
Missing & $0(-)$ & $1(0.5)$ & 0.569 \\
Statins, No. (\%) & $72(38.3)$ & $89(43)$ & -10.8 \\
Yes & $0(-)$ & $3(1.4)$ & 0.057 \\
Missing & $71(37.8)$ & $126(60.9)$ & -58.8 \\
Antiplatelets, No. (\%) & $0(-)$ & $16(7.7)$ & \\
Yes & Missing & & \\
\hline
\end{tabular}

p-values were computed by generalized estimating equations (GEE) model with cluster as group variable

$B M I$ Body Mass Index, BP Blood Pressure, GFR Glomerular Filtrate, HbA1c Glycated haemoglobin, IQR interquartile range, SD standard deviation, ACEi AngiotensinConverting-Enzyme inhibitor, ARBs Angiotensin II receptor blockers

*Metformin, Pioglitazone, acarbose, etc

arm. Global imbalance, estimated using c-statistic, was around 0.8 .

\section{Intervention output}

The median duration of intervention was 3.84 and 3.40 years in MT and SoC group, respectively. At the end of the intervention phase period, we found a significantly lower level of SBP, HbA1c, total and LDL cholesterol in the intensive-treatment arm (Table 2).

In particular, during the Interventional Study Period, we observed a significant decline of BP, HbA1c and lipids in the MT arm already after the first year of intervention, after which mean values remained almost stable; conversely, no significant changes were detected in SoC group (Fig. 2).

In MT group, achievement of BP target $<130 / 80 \mathrm{mmHg}$ was significantly higher overall $(68.3 \%$ vs $50.9 \%, \mathrm{p}<0.001)$ and by component (SBP 77.4\% vs $55.6 \%$, p $<0.001-$ DBP $81.9 \%$ vs $78.1 \%, P=0.003$ ) (Additional file 3: Fig. S2). Similarly, HbA1c, total and LDL cholesterol targets were achieved more frequently in the intensive-treatment arm.

In MT arm, most of patients reached $\geq 4$ targets (53\% vs $29 \%$ in SoC arm); similarly, all six planned targets were more frequently achieved in the intervention arm $(11.1 \%$ vs $2.4 \%)$.

Both hyperkaliemia $\left(\mathrm{K}^{+}>5.5 \mathrm{mEq} / \mathrm{L}\right)$ and renal impairment (eGFR reduction $>30 \%$ than baseline values) were observed more frequently in the MT arm than in SoC arm $[19(10 \%)$ vs. $9(5 \%) ; P=0.120$ and $30(16 \%)$ vs 15 (9\%), $P=0.080$, respectively), likely due to more frequent use of dual blockade of RAS with ARBs and ACEi association. Nonetheless, events leading to permanent ACEi/ ARB or double block discontinuation were infrequent: hyperkaliemia $(8 ; 4.1 \%$ in the MT group vs. $4 ; 2.1 \%$ in the SoC group; $P=0.558)$ and renal impairment $(3 ; 1.6 \%$ in the MT group vs. $2 ; 1.2 \%$ in the SoC group; $P=1.000$ ).

\section{Survival analysis}

During follow-up (median 13.0 years, IQR 12.4-13.3). 262 MACEs were recorded, 146 in the SoC group and 116 in the intensive-therapy group (Table 3). Kaplan Meier analysis (Fig. 3A) showed a major difference between the two arms, with a median survival of 9.6 years $(95 \%$ CI 8.6-10.7) for SoC group and 12.5 years (95\% CI 11.5-13.3) for intensive-therapy group. The Cox shared-frailty model confirmed this finding at univariate analysis (HR 0.49, 95\% CI $0.35-$ $0.69, P<0.001$ ), as after multiple adjustments (adjusted HR 0.47 , 95\% CI $0.30-0.74, P=0.001$ ).

Secondary endpoints are reported in Table 3. Allcause mortality occurred in 189 patients (103 in the SoC group and 86 in the intensive-therapy group). The Kaplan-Meier analysis disclosed a median survival time of 11.9 years (95\% CI 10.5-13.05) for SoC group and 13.5 years (95\% CI 13.3-15.0) for intensivetherapy group (Fig. 3 panel B). The Cox shared-frailty model showed a significant difference both at univariate model (HR 0.58, 95\% CI 0.34-0.98, $P=0.046$ ), and after adjustment for confounding variables (adjusted HR $0.53,95 \%$ CI $0.29-0.93, P=0.027$ ). Incidence of $\mathrm{MI}$ and stroke was lower in intensive-therapy group, while PTCA and major amputation did not differ. These inter-group comparisons were unadjusted because of the small number of events.

As additional secondary endpoint, we also analysed MACEs at the end of intervention phase. Overall, 74 MACEs were recorded, 50 in the SoC group and 24 in the intensive-therapy group (Table 3 ) with an unadjusted HR 0.28 (95\% CI 0.13-0.63; $P=0.002$ ). During intervention phase, we recorded kidney failure (either initiation of chronic dialysis or eGFR $<15 \mathrm{~mL} /$ $\min / 1.73 \mathrm{~m}^{2}$ ) in 12 patients ( 5 in the $\mathrm{SoC}$ group and 7 in the intensive-therapy group). 
Table 2 Differences in demographic, clinical and laboratory parameters and pharmacological treatment between SoC and Intensive therapy group at end of intervention

\begin{tabular}{|c|c|c|c|}
\hline \multirow[t]{2}{*}{ Parameter } & \multicolumn{2}{|l|}{ End of treatment } & \multirow[b]{2}{*}{$p^{* *}$} \\
\hline & SoC Group $(n=169)$ & Intervention group $(n=199)$ & \\
\hline Systolic blood pressure (mmHg) & $135.1(15.2)$ & $127.3(8.7)$ & 0.004 \\
\hline Diastolic blood pressure $(\mathrm{mmHg})$ & $78.8(8.8)$ & $78.1(5.6)$ & 0.110 \\
\hline$B P<130 / 80 \mathrm{mmHg}(\%)$ & $132(74.2)$ & $163(84.5)$ & 0.045 \\
\hline \multicolumn{4}{|l|}{ Laboratory tests } \\
\hline eGFR EPI-CKD (mL/min) & $60.7(22.9)$ & $60.4(22.5)$ & 0.920 \\
\hline Albuminuria (mg/day) & $90.2(38-160)$ & $54(11-180)$ & 0.179 \\
\hline Albuminuria < 30 mg/day (\%) & $18(13.0)$ & $62(37.6)$ & 0.047 \\
\hline Fasting plasma glucose (mg/dL) & $153.6(44.8)$ & $147.4(39.4)$ & 0.199 \\
\hline $\mathrm{HbA1c}(\%)$ & $7.4(1.1)$ & $6.9(0.6)$ & 0.009 \\
\hline $\mathrm{HbAlc}<7 \%(\%)$ & $88(52.1)$ & $129(64.8)$ & 0.133 \\
\hline Total cholesterol (mg/dL) & $190.9(33)$ & $173(28.9)$ & 0.015 \\
\hline Total cholesterol < 175 mg/dL (\%) & $55(32.5)$ & $106(53.2)$ & 0.054 \\
\hline LDL cholesterol (mg/dL) & $122.3(29.8)$ & $100.5(26.5)$ & $<0.001$ \\
\hline$L D L<100 \mathrm{mg} / \mathrm{dL}(\%)$ & $34(20.1)$ & $106(53.2)$ & 0.001 \\
\hline Triglycerides (mg/dL), median (IQR) & $122(90-171)$ & $145(120-169)$ & 0.794 \\
\hline \multicolumn{4}{|l|}{ Therapy } \\
\hline Anti-hypertensive drugs, median (IQR) & $1(1-3)$ & $2(1-3)$ & $<0.001$ \\
\hline \multicolumn{4}{|l|}{ ACEi/ARBs, No. (\%) } \\
\hline ACEi & $104(61.8)$ & $7(3.4)$ & \\
\hline$A R B S$ & $56(33.1)$ & $5(2.3)$ & \\
\hline$A C E+A R B S$ & $3(1.8)$ & $187(94.3)$ & \\
\hline None & $6(3.6)$ & - & $<0.001$ \\
\hline Diuretics, No. (\%) & $84(49.7)$ & $113(56.8)$ & 0.864 \\
\hline Calcium channel Blockers, №. (\%) & $59(34.9)$ & $79(39.7)$ & 0.687 \\
\hline Beta-blockers, No. (\%) & $30(17.8)$ & $38(19.1)$ & 0.961 \\
\hline Alpha-blockers, №. (\%) & $6(3.6)$ & $10(4.8)$ & 0.574 \\
\hline Diabetes therapy, №. (\%) & & & 0.843 \\
\hline Diet & $5(2.9)$ & $5(2.5)$ & \\
\hline Insulin & $57(33.7)$ & $73(36.7)$ & \\
\hline Oral anti-hyperglycemics & $77(45.6)$ & $88(44.2)$ & \\
\hline Combined therapy & $16(9.5)$ & $25(12.6)$ & \\
\hline Missing & $14(8.3)$ & $8(4)$ & \\
\hline Use of statins, No. (\%) & $84(49.7)$ & $110(55.3)$ & 0.991 \\
\hline Use of antiplatelets, No. (\%) & $104(61.6)$ & $148(74.4)$ & 0.905 \\
\hline
\end{tabular}

Data are mean (SD) or median [IQR]

P Blood Pressure, GFR Glomerular Filtration rate, HbA1c glycated haemoglobin, ACEi Angiotensin-Converting-Enzyme inhibitor, ARB Angiotensin II receptor blockers ** P values refer to difference between SoC and Intensive therapy at the end of treatment and were computed by generalized estimating equations (GEE) model with cluster as group and adjusted with baseline value as covariate

\section{Discussion}

This cluster-randomized trial demonstrates that in DKD patients at very high cardiovascular risk, a multifactorial intensive treatment significantly reduces the risk of MACEs versus SoC. This cardiovascular benefit becomes evident early during the three years of intervention and persisted over the long-term follow-up of
13 years. Noteworthy, the favourable effect extends to all-cause mortality.

Several studies have demonstrated the high cardiovascular risk in T2DM. Among these, the large meta-analysis by the Emerging Risk Factors Collaboration centre showed a significantly higher adjusted risk among individuals with diabetes at younger ages and women, as 


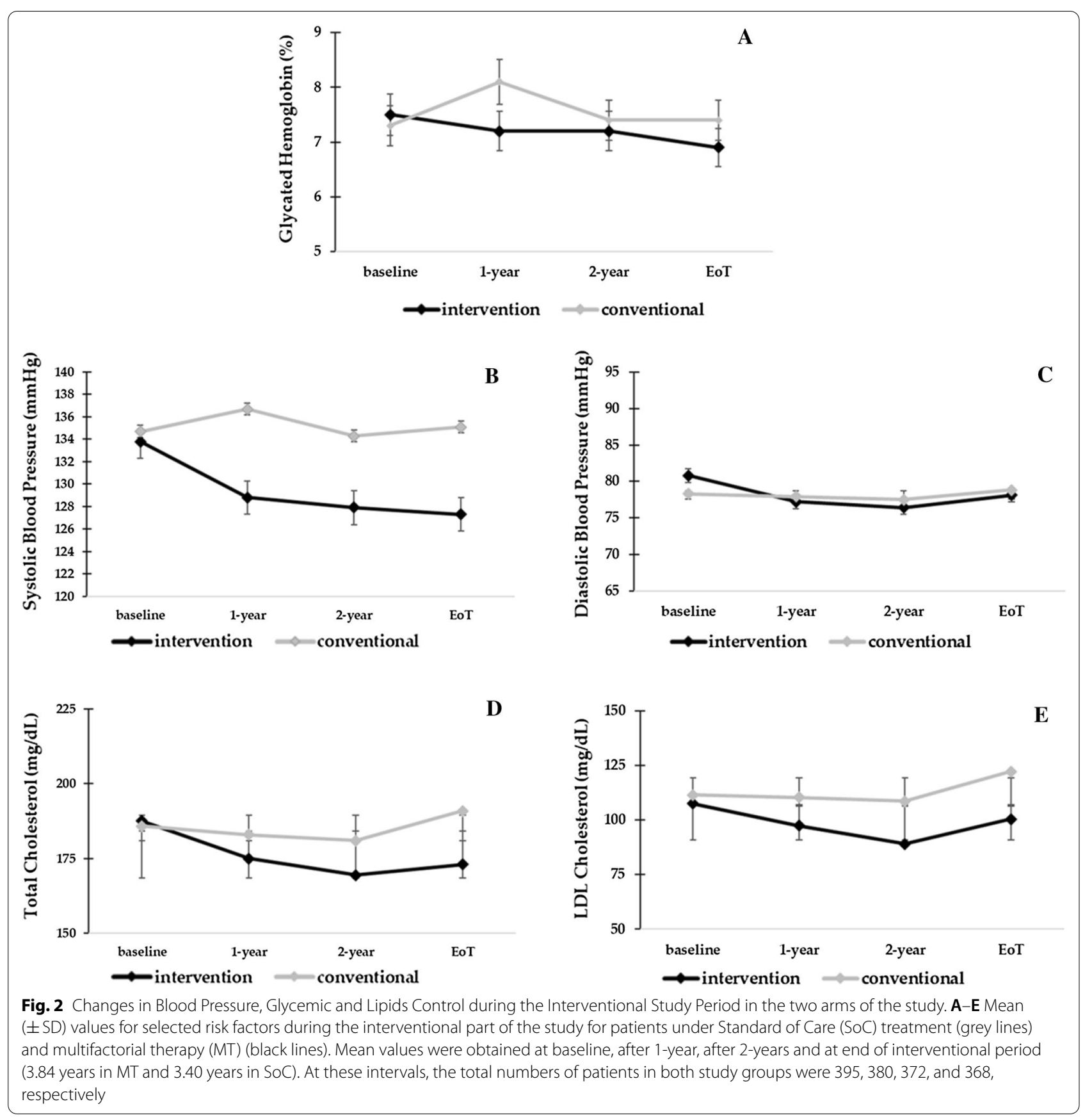

well as a strong association with cardiovascular deaths [23]. Likewise, in the Swedish National Diabetes Register, a close association between CV events and the number of risk factors at goal was further disclosed [24]. This high residual risk is matter of debate. It may be possibly due to inadequate global management of risk factors. Indeed, the European Euro Heart Survey showed how a poly-pharmacological approach improved prognosis of patients with diabetes [25]. Nevertheless, a multi-drug therapy in T2DM does not guarantee for the gain of the different goals suggested by guidelines, as reported by the EUROASPIRE IV survey [26]. Such a gap between goals and real-life findings suggests the need of a multidisciplinary approach in diabetology outpatient clinics [27-30].

Although several observational studies have confirmed the association between risk factors and cardiovascular outcome, solid evidence on the efficacy of a multifactorial intervention in reducing the risk in DKD patients is 
Table 3 Clinical outcome in the two groups $(n=395)$

\begin{tabular}{lllr}
\hline Event & SoC group $(\mathbf{n = 1 8 8})$ & Intervention group $(\mathbf{n}=\mathbf{2 0 7})$ & $\mathbf{p}^{*}$ \\
\hline End of follow-up & & & \\
MACEs, No. (\%) & $146(77.8)$ & $116(56.1)$ & $<6(41.6)$ \\
All-cause death, No. (\%) & $103(54.8)$ & $32(15.5)$ & 0.001 \\
Myocardial infarction, No. (\%) & $44(23.4)$ & $19(9.2)$ & $16(7.8)$ \\
Stroke, No. (\%) & $28(14.9)$ & $2(0.9)$ & 0.046 \\
Revascularization, No. (\%) & $13(6.9)$ & & 0.002 \\
Major amputation, No. (\%) & $3(1.6)$ & $24(11.6)$ & 0.33 \\
Follow-up at end of intervention phase & & 0.002 \\
MACEs, No. (\%) & $50(26.6)$ &
\end{tabular}

MACE Major Adverse Cardiovascular Event

* p-value calculated with univariable Cox shared-frailty model. Multivariable estimates are reported in the manuscript

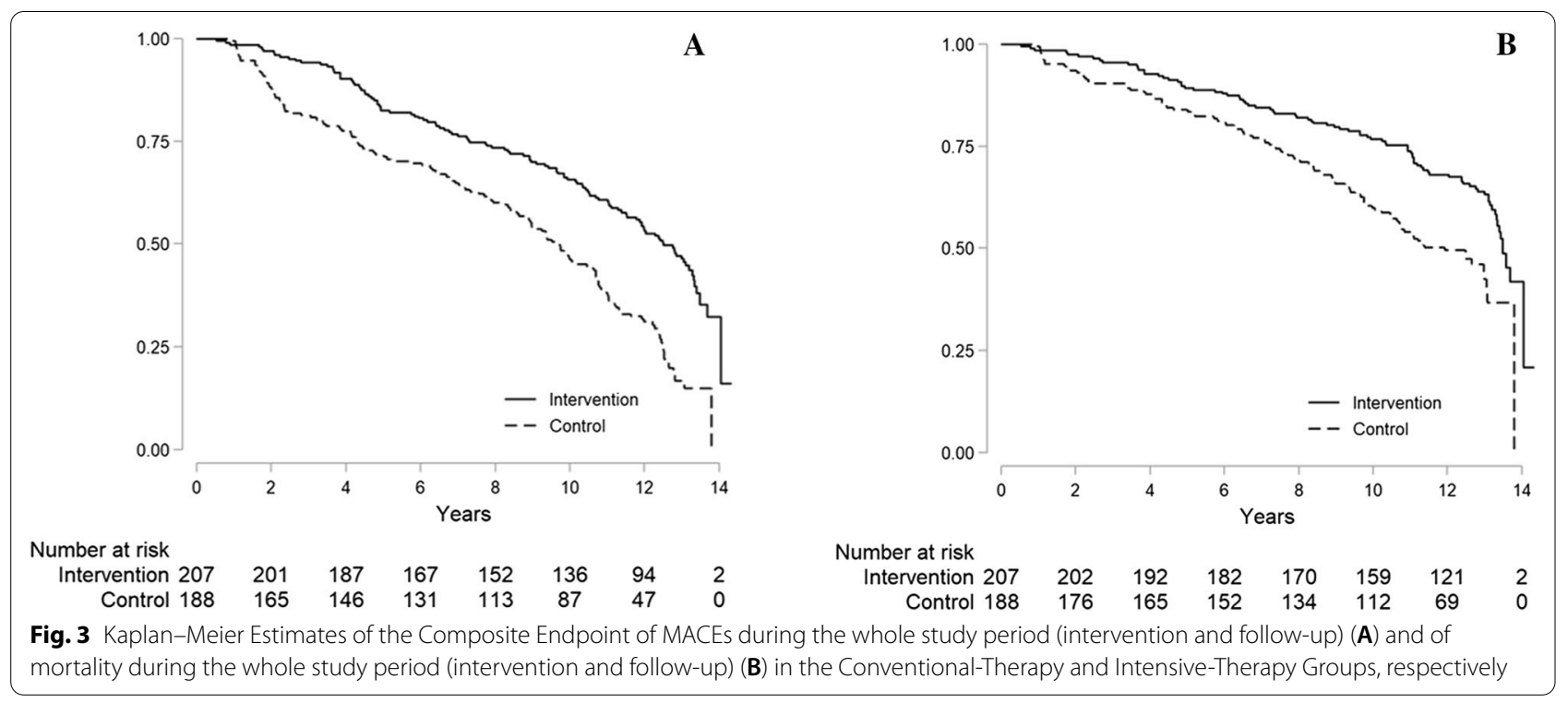

limited. Indeed, only one randomized trial (Steno-2) has addressed this issue.[7, 8]. Steno-2 trial randomized 160 T2DM albuminuric patients either to an intensive multifactorial therapy or SoC. Although main therapeutic goals (HbA1c, lipids and BP) were not fully achieved in the intensive-treatment group, after a mean follow-up of 7.8 years, patients intensively treated had a significant $50 \%$ lower risk of both cardiovascular and microvascular events [7].

To date, the findings of Steno-2 study were not confirmed by other studies, even in those enrolling diabetic subjects regardless of DKD selection criterion. In multicentre, open-label, randomized, J-DOIT3 study [31], type 2 diabetes patients, not specifically selected with DKD, were randomly assigned to either multifactorial intensive or conventional therapy and followed up for a median of 8.5 years. Notably, the study did not evidence any efficacy of intensified multifactorial intervention compared with current standard care on the prevention of a composite of coronary events, cerebrovascular events, and all-cause mortality.

Of note, the present trial was originally conceived to overcome the limits of the Steno-2, in terms of study planning (we adopted a multicentre study design) as well as patients' selection. Indeed, our population was older (67 vs 55 yrs), well balanced for sex (in Steno-2 men accounted for $75 \%$ study population) and with a lower eGFR ( $64 \mathrm{vs} 117 \mathrm{ml} / \mathrm{min} / 1.73 \mathrm{~m}^{2}$ ). These features make our population much closer to the albuminuric T2DM subjects from National Registries, and thus more representative of real-life population with diabetes $[24,32]$. We originally enrolled only patients at primary cardiovascular prevention, unlike Steno-2, which also recruited patients with prior cardiovascular events. Furthermore, our cohort had DR as inclusion criterion. The combination of albuminuria and DR portends a 
higher cardiovascular risk [33]. The higher cardiovascular risk in our study population vs Steno-2 cohort more likely explains the effectiveness of a shorter multifactorial treatment (3.84 vs. 7.8 years in Steno-2). Moreover, in our study, the risk reduction induced by intensive treatment occurred earlier than in Steno-2; indeed, frailty model showed a significantly lower risk for overall mortality and MACEs, already evident at end of intervention phase.

The pathophysiological mechanisms underlying the cardiovascular protective effect in MT arm can only be hypothesized. Besides the well-known effects of BP lowering treatment with RAS inhibition, the intensive glycaemic control with the consequent "legacy effect" on AGEs [33], the effect of statin on LDL and inflammatory cytokines [34,35], and the inhibition of platelet adhesion, which reduces leukocyte infiltration and atherosclerosis [36], more likely, the combination of different interventions may have been critical.

In our study, centres allocated to intensified therapy achieved a higher rate of patients at target for both single and multiple risk factors. Of note, the intervention study took place before GLP1-RA and SGLT2i had been marketed in Italy. Therefore, these drugs, though characterized by important cardioprotective effect [1], could not be assessed.

In the NID-2 study, the intervention phase was completed in 2011, prior to the publication of the Veterans Affairs Nephropathy in Diabetes (VA-NEPHRON-D) study, [37] which firstly showed in diabetic nephropathic patients an association between the combination of ACEi and ARB and an increased risk either of decreased eGFR and/or of the onset of hyperkaliaemia. However, the NID-2 protocol required a strict monitoring of renal function and serum electrolytes and required the permanent withdrawal of dual RAS blockade in the case of persistent side effects, as above reported. Notably, during intervention phase the percentage of subjects who had to suspend treatment with drugs active on the RAS due to persistent side effects was low and was similar to what was recorded in the Altitude study [38].

Our study has several strengths. First, it is the first multicentre study showing the effectiveness of a multifactorial treatment on MACEs. Second, durability of our findings was confirmed by means of a long follow-up. In addition, randomization by centre makes the study closer to real-life clinical practice. On this regard, it is noteworthy that the SoC arm showed a more favourable clinical picture at baseline in comparison with the intervention arm. This finding reasonably excludes the possibility of selecting in SoC arm those physicians with lower attitude to adhere to clinical guidelines. However, the cluster-randomized design has a number of limitations, such as the lack of blind assignment, power and precision are lower than an individually randomized trial and the ability to control for both known and unknown confounder is reduced. There is extensive discussion in the Literature on strategies and methods to reduce these limitations and mitigate the impact on results. We attempted at the best use of them to achieve internal validity so that unbiased estimates of efficacy can be obtained within the study population and generalized to target population [39]. Specifically, the main results of the study were adjusted for both the cluster factor due to randomization and for the main variables resulted either unbalanced or clinically and statistically associated with outcome.

As major limitation, we did not collect clinical and laboratory data during the follow-up occurring after intervention phase but only events of interest (death and cardiovascular events) thus precluding the possibility of identify a prevailing factor definitely associated with risk reduction. In addition, some factors possibly related to CV outcome, such as uric acid or proBNP [40, 41], were not included in our laboratory panel. Therefore, we cannot identify one or more specific factor definitely associated with risk reduction; however, our original aim was to test efficacy of a global approach rather than effects of single interventions.

Moreover, the choice of a composite of fatal and nonfatal MACEs as primary endpoint may explain the lack of a sufficient power for mortality outcome. Finally, the SoC population was two-years older than the intensified treatment one. However, considering the mean age in both groups $>65$ years, the high CV risk as inclusion criterion, and both a higher mean DBP and a much lower prevalence of HbA1c target subjects at baseline in the intervention group, we believe the two groups are clinically comparable.

\section{Conclusions}

Therefore, this multicentre trial demonstrates that a strategy based on intensive treatment of main risk factors is more effective than standard of care in preventing MACEs in type 2 DKD. Pursuing such a strategy triggers a marked improvement of outcomes occurring early after its implementation and lasting in the long-term.

\footnotetext{
Abbreviations

ACEi: Angiotensin converting enzyme inhibitor; ARBs: Angiotensin II receptor blockers; CRF: Case Report Form; DKD: Diabetic kidney disease; DR: Diabetic retinopathy; DBP: Diastolic blood pressure; GEE: Generalized estimating equations; HbA1c: Glycated haemoglobin; HR: Hazard Ratio; MACEs: Major fatal and non-fatal cardiovascular events; MT: Multifactorial intensive therapy; MI: Myocardial infarction; NID-2: Nephropathy in diabetes type 2; RAS: Reninangiotensin system; PTCA: Revascularization procedures; SDiff: Standardized differences; SoC: Standard-of-Care; SBP: Systolic blood pressure; T2DM: Type 2 diabetes mellitus.
} 


\section{Supplementary Information}

The online version contains supplementary material available at https://doi. org/10.1186/s12933-021-01343-1.

Additional file 1: Appendix S1. Intervention by randomization.

Additional file 2: Figure S1. Standardized Differences for Imbalance Detection. The standardized differences are reported on $\mathrm{x}$ - axis. The type of point describes the imbalance in favor of the treatment arm. The right panel shows the only variables for which a therapeutic achievement target has been calculated. Covariance Imbalance Detection: if SDiff was either $\geq 5 \%$ or $<5 \%$ but its correlation with at least one covariate with a SDiff $\geq 5$ was greater or equal to 0.2 .

Additional file 3: Figure S2. Targets Achievement at the end of intervention phase. Type (left panel) and number (right panel) of target for single cardiovascular risk factors achieved at the end of intervention period in conventional therapy (black bars) vs Intensive therapy (gray bars).

\section{Acknowledgements}

Dr. Raffaele Galiero, Dr. Pia Clara Pafundi and Dr. Vittorio Simeon were supported by the Programma VALERE, University of Campania "Luigi Vanvitelli".

NID-2 Study Group Investigators: U. Amelia, C. Acierno, P. Calatola, O. Carbonara, A. Caturano, G. Conte, G. Corigliano, M. Corigliano, R. D'Urso, A. De Matteo, L. De Nicola, N. De Rosa, E. Del Vecchio, G. Di Giovanni, A. Gatti, S. Gentile, L. Gesuè, L. Improta, A. Lampitella Jr, A. Lampitella, A. Lanzilli, N. Lascar, S. Masi, P. Mattei, V. Mastrilli, P. Memoli, R. Minutolo, R. Nasti, A. Pagano, M. Pentangelo, E. Pisa, E. Rossi, F.C. Sasso, S. Sorrentino, R. Torella, R. Troise, P. Trucillo, A. A. Turco, S. Turco, F. Zibella, L. Zirpoli.

Declaration of Transparency: The lead author affirms that this manuscript is an honest, accurate, and transparent account of the study being reported; that no important aspects of the study have been omitted; and that any discrepancies from the study as planned (and, if relevant, registered) have been explained.

\section{Authors' contributions}

Concept and design: FCS, LDN, RMi. Acquisition of data: PCP, RG, RN, LR. Data analysis: VS, PCP, PC. Interpretation of data: FCS, PCP, LDN, RMi, PC, VS, LEA, TS, RMa. Drafting of the manuscript: FCS, PCP, PC, LDN, RMi, VS. Critical revision of the manuscript for important intellectual content: FCS, LDN, RMi, PC. All authors read and approved the final manuscript.

\section{Funding}

The study was funded by Ministero dell'Università e della Ricerca, PRIN 2007.

\section{Availability of data and materials}

All data generated or analyzed during this study are included in this published article and its supplementary information files.

\section{Declarations}

\section{Ethical approval and consent for participate}

The trial was conducted in accordance with the guiding principles of the Declaration of Helsinki and was approved by the local ethics committee.

\section{Consent for publication}

Not applicable.

\section{Competing interests}

Luca De Nicola has received fees for scientific consultation and/or lectures by Astellas, AstraZeneca, Mundibiopharma and Vifor Pharma. Roberto Minutolo has been member of Advisory Boards for Astellas, and invited speaker at meetings supported by Amgen, Vifor Pharma. Ferdinando Carlo Sasso has been member of Advisory Boards for Boehringer and for Ely-Lilly and has received fees for scientific consultation and/or lectures by Jansen, Roche Diagnostics, Novo Nordisk, Sanofi, MSD, Astrazeneca. Pia Clara Pafundi, Vittorio Simeon, Paolo Chiodini, Raffaele Galiero, Luca Rinaldi, Riccardo Nevola, Teresa Salvatore, Raffaele Marfella, Celestino Sardu, and Luigi Elio Adinolfi have no conflict of interest to disclose.

\section{Author details}

${ }^{1}$ Department of Advanced Medical and Surgical Sciences, University of Campania "Luigi Vanvitelli", Piazza Luigi Miraglia 2, 80138 Naples, Italy. ${ }^{2}$ Medical Statistics Unit, Department of Physical and Mental Health and Preventive Medicine, University of Campania "Luigi Vanvitelli", Piazza Luigi Miraglia 2, 80138 Naples, Italy. ${ }^{3}$ Department of Precision Medicine, University of Campania "Luigi Vanvitelli", Via De Crecchio 7, 80138 Naples, Italy.

Received: 16 May 2021 Accepted: 9 July 2021

Published online: 16 July 2021

\section{References}

1. Rawshani A, Rawshani A, Franzén S, et al. Risk factors, mortality, and cardiovascular outcomes in patients with type 2 diabetes. N Engl J Med. 2018;379(7):633-44. https://doi.org/10.1056/NEJMoa1800256.

2. Fox CS, Matsushita K, Woodward M, et al. Chronic Kidney Disease Prognosis Consortium Associations of kidney disease measures with mortality and end-stage renal disease in individuals with and without diabetes: a meta-analysis. Lancet. 2012;380(9854):1662-73. https://doi.org/10.1016/ S0140-6736(12)61350-6.

3. Tonelli M, Muntner P, Lloyd A, et al. Alberta Kidney Disease Network Risk of coronary events in people with chronic kidney disease compared with those with diabetes: a population-level cohort study. Lancet. 2012;380(9844):807-14. https://doi.org/10.1016/S0140-6736(12)60572-8.

4. Sarnak MJ, Levey AS, Schoolwerth AC, et al. American Heart Association Councils on Kidney in Cardiovascular Disease, High Blood Pressure Research, Clinical Cardiology, and Epidemiology and Prevention. Kidney disease as a risk factor for development of cardiovascular disease: a statement from the American Heart Association Councils on Kidney in Cardiovascular Disease, High Blood Pressure Research, Clinical Cardiology, and Epidemiology and Prevention. Circulation. 2003;108(17):2154-69. https://doi.org/10.1161/01.CIR.0000095676.90936.80.

5. Provenzano M, Coppolino G, De Nicola L, et al. Unraveling cardiovascular risk in renal patients: a new take on old tale. Front Cell Dev Biol. 2019;3(7):314. https://doi.org/10.3389/fcell.2019.00314.

6. Minutolo R, Gabbai FB, Provenzano M, et al. Cardiorenal prognosis by residual proteinuria level in diabetic chronic kidney disease: pooled analysis of four cohort studies. Nephrol Dial Transplant. 2018;33(11):19429. https://doi.org/10.1093/ndt/gfy032.

7. Gaede P, Vedel P, Larsen N, Jensen GV, Parving HH, Pedersen O. Multifactorial intervention and cardiovascular disease in patients with type 2 diabetes. N Engl J Med. 2003;348(5):383-93. https://doi.org/10.1056/ NEJMoa021778.

8. Gaede $\mathrm{P}$, Lund-Andersen $\mathrm{H}$, Parving $\mathrm{HH}$, Pedersen O. Effect of a multifactorial intervention on mortality in type 2 diabetes. N Engl J Med. 2008;358(6):580-91. https://doi.org/10.1056/NEJMoa0706245.

9. Guo VY, Cao B, Wu X, Lee JJW, Zee BC. Prospective association between diabetic retinopathy and cardiovascular disease-a systematic review and meta-analysis of cohort studies. J Stroke Cerebrovasc Dis. 2016;25(7):1688-95. https://doi.org/10.1016/j.jstrokecerebrovasdis.2016. 03.009 .

10. Sasso FC, De Nicola L, Carbonara O, et al. Cardiovascular risk factors and disease management in type 2 diabetic patients with diabetic nephropathy. Diabetes Care. 2006;29(3):498-503. https://doi.org/10.2337/diacare. 29.03.06.dc05-1776.

11. American Diabetes Association. Standards of medical care in diabetes. Diabetes Care. 2005;28(Suppl 1):S4-36.

12. Associazione Medici Diabetologi (AMD) - Società Italiana di Diabetologia (SID) - Standard italiani per la cura del diabete mellito 2005.

13. European Society of Hypertension-European Society of Cardiology Guidelines Committee. 2003 European Society of Hypertension-European Society of Cardiology guidelines for the management of arterial hypertension. J Hypertens. 2003;21(6):1011-53. https://doi.org/10.1097/ 00004872-200306000-00001.

14. de Backer G, Ambrosioni E, Borch-Johnsen K, et al. Third Joint Task Force of European and Other Societies on Cardiovascular Disease Prevention in Clinical Practice. European guidelines on cardiovascular disease prevention in clinical practice. Third Joint Task Force of European and 
Other Societies on Cardiovascular Disease Prevention in Clinical Practice. Eur Heart J. 2003;24(17):1601-10. https://doi.org/10.1016/s0195-668x(03) 00347-6.

15. Powers WJ, Rabinstein AA, Ackerson T, et al. Guidelines for the early management of patients with acute Ischemic stroke: a guideline for healthcare professionals from the American Heart Association/American Stroke Association [published correction appears in Stroke. 2018;49(3):e138. https://doi.org/10.1161/STR.0000000000000158]

16. Ponikowski P, Voors AA, Anker SD, et al. ESC Guidelines for the diagnosis and treatment of acute and chronic heart failure: the task force for the diagnosis and treatment of acute and chronic heart failure of the European Society of Cardiology (ESC) Developed with the special contribution of the Heart Failure Association (HFA) of the ESC [published correction appears in Eur Heart J. 2016. Eur Heart J. 2016;37(27):2129-2200. https://doi.org/10.1093/eurheartj/ehw128.

17. Thygesen K, Alpert JS, Jaffe AS, et al. ESC Scientific Document Group. Fourth universal definition of myocardial infarction. Eur Heart $J$. 2019;40:237-69. https://doi.org/10.1093/eurheartj/ehy462.

18. Wilkinson C, Ferris FL, Klein RE, et al. Proposed international clinical diabetic retinopathy and diabetic macular edema disease severity scales. Ophthalmology. 2003;110(9):1677-82. https://doi.org/10.1016/s01616420(03)00475-5.

19. Skali H, Uno H, Levey AS, Inker LA, Pfeffer MA, Solomon SD. Prognostic assessment of estimated glomerular filtration rate by the new Chronic Kidney Disease Epidemiology Collaboration equation in comparison with the Modification of Diet in Renal Disease Study equation. Am Heart J. 2011;162:548-54. https://doi.org/10.1016/j.ahj.2011.06.006.

20. Leyrat C, Caille A, Foucher Y, Giraudeau B. Propensity score to detect baseline imbalance in cluster randomized trials: the role of the c-statistic. BMC Med Res Methodol. 2016;22(16):9. https://doi.org/10.1186/ s12874-015-0100-4.

21. Hubbard AE, Ahern J, Fleischer NL, et al. To GEE or not to GEE: comparing population average and mixed models for estimating the associations between neighborhood risk factors and health. Epidemiology. 2010;21(4):467-74. https://doi.org/10.1097/EDE.0b013e3181 caeb90.

22. Sasso FC, Chiodini P, Carbonara O, et al. High cardiovascular risk in patients with Type 2 diabetic nephropathy: the predictive role of albuminuria and glomerular filtration rate. The NID-2 Prospective Cohort Study. Nephrol Dial Transplant. 2012;27(6):2269-74. https://doi.org/10.1093/ndt/ gfr644.

23. Seshasai S, Kaptoge S, Thompson A, et al. Emerging Risk Factors Collaboration Diabetes mellitus, fasting glucose, and risk of cause-specific death. N Engl J Med. 2011;364(9):829-41. https://doi.org/10.1056/NEJMoa1008 862.

24. Svensson MK, Cederholm J, Eliasson B, Zethelius B, Gudbjörnsdottir S. Swedish National Diabetes Register Albuminuria and renal function as predictors of cardiovascular events and mortality in a general population of patients with type 2 diabetes: a nationwide observational study from the Swedish National Diabetes Register. Diab Vasc Dis Res. 2013;10(6):520-9. https://doi.org/10.1177/1479164113500798.

25. Anselmino M, Malmberg K, Ohrvik J, Rydén L. Euro Heart Survey Investigators Evidence-based medication and revascularization: powerful tools in the management of patients with diabetes and coronary artery disease: a report from the Euro Heart Survey on diabetes and the heart. Eur J Cardiovasc Prev Rehabil. 2008;15(2):216-23. https://doi.org/10.1097/ HJR.0b013e3282f335d0

26. Gyberg V, De Bacquer D, De Backer G, et al., EUROASPIRE Investigators. Patients with coronary artery disease and diabetes need improved management: a report from the EUROASPIRE IV survey: a registry from the EuroObservational Research Programme of the European Society of Cardiology. Cardiovasc Diabetol. 2015;14:133. https://doi.org/10.1186/ s12933-015-0296-y.

27. Minutolo R, Sasso FC, Chiodini P, et al. Management of cardiovascular risk factors in advanced type 2 diabetic nephropathy: a comparative analysis in nephrology, diabetology and primary care settings. J Hypertens. 2006;24(8):1655-61. https://doi.org/10.1097/01.hjh.0000239303.93872.31.

28. Sasso FC, Rinaldi L, Lascar N, Marrone A, Pafundi PC, Adinolfi LE, Marfella R. Role of Tight Glycemic Control during Acute Coronary Syndrome on CV Outcome in Type 2 Diabetes. J Diabetes Res. 2018;4(2018):3106056. https://doi.org/10.1155/2018/3106056.

29. Sasso FC, Pafundi PC, Gelso A, Bono V, Costagliola C, Marfella R, Sardu C, Rinaldi L, Galiero R, Acierno C, et al., NO BLIND Study Group. Telemedicine for screening diabetic retinopathy: The NO BLIND Italian multicenter study. Diabetes Metab Res Rev. 2019;35(3):e3113. https://doi.org/10.1002/ dmr.3113.

30. Sasso FC, Pafundi PC, Marfella R, Calabrò P, Piscione F, Furbatto F, Esposito G, Galiero R, Gragnano F, Rinaldi L, et al. Adiponectin and insulin resistance are related to restenosis and overall new PCl in subjects with normal glucose tolerance: the prospective AIRE Study. Cardiovasc Diabetol. 2019;18(1):24. https://doi.org/10.1186/s12933-019-0826-0.

31. Ueki K, Sasako T, Okazaki Y, et al. Effect of an intensified multifactorial intervention on cardiovascular outcomes and mortality in type 2 diabetes (J-DOIT3): an open-label, randomised controlled trial. Lancet Diabetes Endocrinol. 2017;5(12):951-64. https://doi.org/10.1016/S2213-8587(17) 30327-3.

32. Brownrigg JR, Hughes $\mathrm{CO}$, Burleigh $\mathrm{D}$, et al. Microvascular disease and risk of cardiovascular events among individuals with type 2 diabetes: a population-level cohort study. Lancet Diabetes Endocrinol. 2016;4(7):588-97. https://doi.org/10.1016/S2213-8587(16)30057-2.

33. Holman RR, Paul SK, Bethel MA, Matthews DR, Neil HAW. 10-Year follow-up of intensive glucose control in type 2 diabetes. N Engl J Med. 2008;359:1577-89. https://doi.org/10.1056/NEJMoa0806470.

34. Hansson GK. Inflammation, atherosclerosis, and coronary artery disease N Engl J Med. 2005;352(16):1685-95. https://doi.org/10.1056/NEJMr a043430.

35. Bohula EA, Giugliano RP, Cannon CP, et al. Achievement of dual lowdensity lipoprotein cholesterol and high-sensitivity C-reactive protein targets more frequent with the addition of ezetimibe to simvastatin and associated with better outcomes in IMPROVE-IT. Circulation. 2015;132(13):1224-33. https://doi.org/10.1161/CIRCULATIONAHA.115. 018381.

36. Massberg S, Brand K, Grüner S, et al. A critical role of platelet adhesion in the initiation of atherosclerotic lesion formation. J Exp Med. 2002;196(7):887-96. https://doi.org/10.1084/jem.20012044.

37. Fried LF, Emanuele N, Zhang JH, et al. VA NEPHRON-D Investigators. Combined angiotensin inhibition for the treatment of diabetic nephropathy. N Engl J Med. 2013;369(20):1892-903. https://doi.org/10.1056/NEJMo a1303154.

38. Parving HH, Brenner BM, McMurray JJ, et al. Cardiorenal end points in a trial of aliskiren for type 2 diabetes. N Engl J Med. 2012;367(23):2204-13. https://doi.org/10.1056/NEJMoa1208799.

39. Hayes R, Moulton L. Cluster randomised trials. Boca Raton, FL: Chapman and Hall/CRC Press; 2009.

40. Li Q, Yang Z, Lu B, et al. Serum uric acid level and its association with metabolic syndrome and carotid atherosclerosis in patients with type 2 diabetes. Cardiovasc Diabetol. 2011;10:72. https://doi.org/10.1186/ 1475-2840-10-72.

41. von Scholten BJ, Reinhard H, Hansen TW, et al. Additive prognostic value of plasma $\mathrm{N}$-terminal pro-brain natriuretic peptide and coronary artery calcification for cardiovascular events and mortality in asymptomatic patients with type 2 diabetes. Cardiovasc Diabetol. 2015;14:59. https:// doi.org/10.1186/s12933-015-0225-0.

\section{Publisher's Note}

Springer Nature remains neutral with regard to jurisdictional claims in published maps and institutional affiliations. 